MATHEMATICS OF COMPUTATION

Volume 66, Number 218, April 1997, Pages 833-839

S 0025-5718(97)00821-1

\title{
RATIONAL EIGENVECTORS IN SPACES OF TERNARY FORMS
}

\author{
LARRY LEHMAN
}

\begin{abstract}
We describe the explicit computation of linear combinations of ternary quadratic forms which are eigenvectors, with rational eigenvalues, under all Hecke operators. We use this process to construct, for each elliptic curve $E$ of rank zero and conductor $N<2000$ for which $N$ or $N / 4$ is squarefree, a weight $3 / 2$ cusp form which is (potentially) a preimage of the weight two newform $\phi_{E}$ under the Shimura correspondence.
\end{abstract}

\section{INTRODUCTION}

If $E$ is a modular elliptic curve over $\mathbb{Q}$ with $\phi_{E}$ its associated weight two newform, an interesting problem is to construct explicitly a weight $3 / 2$ cusp form which is sent to $\phi_{E}$ by the Shimura correspondence. Tunnell [19], Frey [8], Bungert [3], and the author [12] have used such cusp forms to provide information about the group of rational points on twists of specific elliptic curves. (Koblitz uses Tunnell's result as the motivation for an introduction to elliptic curves and modular forms, particularly those of half-integral weight, and we refer to his book [10] for more details on this problem.)

One method of constructing weight $3 / 2$ modular forms, which the author employed in [12], uses quadratic forms in three variables. In [13], we demonstrated a practical method for finding all ternary quadratic forms associated with modular forms of a given level. In this article, we consider further the connection between quadratic forms, modular forms, and elliptic curves from the computational viewpoint. Our main result is the following: For every isogeny class of elliptic curves $E$ having rank zero and conductor $N<2000$ such that either $N$ or $N / 4$ is squarefree, there is an explicitly computed weight $3 / 2$ cusp form, arising from the ternary quadratic forms in a genus which depends on invariants of $E$, which is a candidate for being a preimage of $\phi_{E}$ under the Shimura correspondence. (See Theorem 1 below and the remarks which follow it for a more precise expression of this result.)

\section{HECKE OPERATORS ON TERNARY FORMS AND MODULAR FORMS}

In this section, we briefly recall the necessary background on ternary quadratic forms and their associated modular forms. We refer to a positive definite quadratic form $f(x, y, z)=a x^{2}+b y^{2}+c z^{2}+r y z+s x z+t x y$ whose coefficients are integers having no common divisors as a ternary form. The matrix of $f$ is $A=A_{f}=$ $\left[\begin{array}{ccc}2 a & t & s \\ t & 2 b & r \\ s & r & 2 c\end{array}\right]$. Following the conventions of [13], we define the discriminant of $f$ to

Received by the editor January 17, 1995 and, in revised form, February 7, 1996.

1991 Mathematics Subject Classification. Primary 11E45; Secondary 11F37, 11G05.

Key words and phrases. Quadratic forms, modular forms, elliptic curves.

(C)1997 American Mathematical Society 
be $d=\operatorname{det}(A) / 2$ and its level to be the unique integer $N$ such that $N A^{-1}$ is the matrix of a ternary form. Ternary forms $f$ and $g$ are said to be equivalent over a ring $R$ if there is an $R$-unimodular matrix $U$ (a matrix with entries in $R$ whose determinant is a unit in $R$ ) such that $A_{g}=U A_{f} U^{t}$. Two ternary forms are in the same class if they are equivalent over $\mathbb{Z}$. They are in the same genus if they are equivalent over $\mathbb{Z}_{p}$ for all primes $p$ and over the real numbers. This is the case if and only if the ternary forms have the same discriminant and level and the same collection of genus symbols. (See [13, p. 410] for the definition of genus symbols.)

Now consider a genus of ternary forms of level $N$, with $G=\left\{f_{1}, \ldots, f_{n}\right\}$ as class representatives. Let $\mathcal{M}$ be the complex vector space having $G$ as a basis. Then there is a commutative family of linear operators on $\mathcal{M}, \mathcal{T}=\left\{T_{p} \mid p\right.$ prime, $\left.p \nmid N\right\}$, which we refer to as Hecke operators. We can define $T_{p}$ as follows (see [1] for more details and references): For each $f \in G$, the congruence $f(x, y, z) \equiv 0(\bmod p)$ is a nonsingular conic over $\mathbb{F}_{p}$, call it $S_{p}(f)$, and so contains $p+1$ points. For each point $P$ on $S_{p}(f)$, there is a $\mathbb{Z}$-unimodular matrix $U$ such that the first row of $U$, viewed as a point in projective space over $\mathbb{F}_{p}$, is the same as $P$, and such that $U A_{f} U^{t}=\left[\begin{array}{ccc}2 p^{2} a & p t & s \\ p t & 2 b & r \\ s & r & 2 c\end{array}\right]$ with $a, t \in \mathbb{Z}$. Then let $A_{g}=\left[\begin{array}{ccc}2 a & t & s \\ t & 2 b & p r \\ s & p r & 2 p^{2} c\end{array}\right]$ be the matrix of a ternary form $g$. One can show that $f$ and $g$ are in the same genus and that the class of $g$ depends only on the point $P$ and the class of $f$. Denote the class of $g$ by $P(f)$. So now we can define $T_{p}$ by setting $T_{p}\left(f_{i}\right)=\sum_{P \in S_{p}\left(f_{i}\right)} P\left(f_{i}\right)$ for $1 \leq i \leq n$, and extending $T_{p}$ to $\mathcal{M}$ by linearity.

We are interested in finding rational eigenvectors in $\mathcal{M}$, that is, eigenvectors which have rational eigenvalues under each of the Hecke operators. We note some useful facts about eigenvectors in the following two propositions.

Proposition 1. Let $g=\sum_{i=1}^{n} \alpha_{i} f_{i}$ be an eigenvector of $T_{p}$ (for some prime $p \nmid N$ as above) with eigenvalue $\lambda$. Then $\lambda$ is an algebraic integer which is rational if each $\alpha_{i}$ is rational. Furthermore $|\lambda| \leq p+1$ and $\lambda=p+1$ if $\sum_{i=1}^{n} \alpha_{i} \neq 0$.

Proof. If $B=\left[b_{i j}\right]$ is the representation of $T_{p}$ with respect to the basis $G$, then each $b_{i j}$ is a nonnegative integer and for all $j, \sum_{i=1}^{n} b_{i j}=p+1$. The first statement in Proposition 1 is then obvious. For the second, consider the sums $\sum_{i=1}^{n}\left|\lambda \alpha_{i}\right|$ and $\sum_{i=1}^{n} \lambda \alpha_{i}$.

We will denote the subspace of $\mathcal{M}$ consisting of all $\sum_{i=1}^{n} \alpha_{i} f_{i}$ with $\sum_{i=1}^{n} \alpha_{i}=0$ as $\mathcal{S}$ and refer to these as cusp vectors in $\mathcal{M}$. It is easy to see that $\mathcal{S}$ is invariant under all $T_{p}$. The fact that the Hecke operators in $\mathcal{T}$ commute allows us to conclude the following:

Proposition 2. Let $V$ be a subspace of $\mathcal{M}$ which is invariant under all operators in $\mathcal{T}$. For a specific prime $p$ and some $\lambda \in \mathbb{C}$, let $W$ be the $\lambda$-eigenspace of $T_{p}$ in $V$. Then $W$ is also invariant under $\mathcal{T}$.

Proof. If $w$ is any element in $W$, then for any prime $q, T_{p}\left(T_{q}(w)\right)=T_{q}\left(T_{p}(w)\right)=$ $T_{q}(\lambda w)=\lambda T_{q}(w)$. Thus $T_{q}(w) \in W$ by definition.

If $f$ is a ternary form with level $N$ and discriminant $d$, then the complex function on the upper half-plane defined by

$$
\theta_{f}(z)=\sum_{(k, l, m) \in \mathbb{Z}^{3}} e^{2 \pi i \cdot f(k, l, m) z}=\sum_{n=0}^{\infty} a_{n} q^{n}, \text { where } q=e^{2 \pi i z},
$$


is a modular form of weight $3 / 2$, level $N$, and character $\chi_{d}[17]$. The latter expression is called the $q$-expansion of $\theta_{f}$. Modular forms of the same weight, level, and character are equal if their $q$-expansions are identical for sufficiently many terms, depending on the level of the forms [15]. For any prime $p$, there is a Hecke operator $T\left(p^{2}\right)$ which takes a form $\theta_{f}$ to another modular form of the same weight, level, and character. For $p \mid N$, we can define $T\left(p^{2}\right)$ in terms of the $q$-expansion of $\theta_{f}$ by $T\left(p^{2}\right) \theta_{f}=\sum_{n=0}^{\infty} a_{p^{2} n} q^{n}$. (See [10] for the more general definition of Hecke operators, and for more details on these terms.)

If $\mathcal{M}$ is spanned by a genus $G=\left\{f_{1}, \ldots, f_{n}\right\}$ of ternary forms of level $N$, then there is a linear map, which we will denote by $\Theta$, from $\mathcal{M}$ into a space of modular forms, determined by sending each ternary form $f \in G$ to the modular form $\theta_{f} / 2$. We will denote the image of $\mathcal{M}$ as $M$ in general. Because $\theta_{f}-\theta_{g}$ is a cusp form if $f$ and $g$ are in the same genus [16], it is easy to see that the image of $\mathcal{S}$ under $\Theta$, call it $S$, is the subspace of cusp forms in $M$. Results of Eichler [7] and Ponomarev [14] (see also [3, Prop. 4]) indicate that the Hecke operators $T_{p}$ defined on $\mathcal{M}$ are essentially the same as $T\left(p^{2}\right)$ on $M$, that is, for primes $p \nmid N$ that $\Theta \circ T_{p}=T\left(p^{2}\right) \circ \Theta$. In particular, an eigenvector in $\mathcal{M}$ is mapped to an eigenform in $M$ (or to zero, as noted in Theorem 1 below).

\section{Rational EigenVECTORS ASSOCIATED to ELLiPTIC CURVES}

We now note some connections between certain elliptic curves and eigenvectors in specific spaces of ternary forms. Let $E$ be a representative of an isogeny class of modular elliptic curves over $\mathbb{Q}$ and let $\phi_{E}$ be its associated weight two newform. Suppose that $E$ has even rank and that its conductor is of the form $N=2^{e} Q$ where $Q$ is an odd, squarefree integer and $0 \leq e \leq 3$. For each prime $p \nmid N$, let $\lambda_{p}$ be the eigenvalue of $\phi_{E}$ under the Hecke operator $T(p)$. For $p \mid N$, let $\varepsilon_{p}$ be the eigenvalue of $\phi_{E}$ under the $W_{p}$ involution. Define a squarefree integer $\gamma$ by saying that $p \mid \gamma$ if and only if $\varepsilon_{p}=-1$. We will say that $\gamma$ is the genus number associated to the collection $\left\{\varepsilon_{p}\right\}$ (or to $\phi_{E}$ or to $E$ ). Since $E$ has even rank, it is conjectured that $\prod \varepsilon_{p}=-1$ or equivalently that $\gamma$ has an odd number of prime factors. (See [18] for more details on these operators.)

To $E$, we associate a genus of ternary forms in the following way. Let the level of the genus equal $2^{r} Q$ and the discriminant equal $2^{s} Q^{2}$ where $r$ and $s$ are as follows: If $e=0$, then $r=2$ and $s=4$; if $e=1$, then $r=2$ and $s=2$; if $e=2$, then $r=3$ and $s=4$; and if $e=3$, then $r=4$ and $s=5$. For a ternary form $f$ of this level and discriminant, the relevant genus symbols are $(f / p)$ for all $p \mid Q$, and if $e=0$, $(f / 4)$ (but in that case, $(f / 4)$ must be -1$)[13$, Prop. 5]. In each case, choose $(f / p)$ to be $\varepsilon_{p}\left(-2^{s} / p\right)$. Let $\mathcal{M}(N, \gamma)$ be the space spanned by all classes of ternary forms in the genus having level, discriminant, and genus symbols as defined here (with $\mathcal{S}(N, \gamma)$ its subspace of cusp vectors, and so forth).

In the case in which $e=0$, the fact that $(f / 4)=-1$ implies that any cusp form in $S(N, \gamma)$ has the form $\sum_{n=1}^{\infty} a_{n} q^{n}$, where $a_{n}=0$ if $n \equiv 1$ or $2(\bmod 4)$. Thus $S(N, \gamma)$ is a subspace of the space denoted as $S_{3 / 2}(N)$ in [11]. In general, this space is not invariant under the operator $T\left(2^{2}\right)$ as defined above, but Kohnen defines a revised operator, which we will denote by $T^{\prime}\left(2^{2}\right)$, for this space (see [11, p. 42] for the definition of this operator).

We can now state our main result as follows: 
Theorem 1. For every isogeny class of elliptic curves $E$ of even rank and conductor $N=2^{e} Q<2000$, with genus number $\gamma$, there is an explicitly computed rational eigenvector $g \in \mathcal{M}(N, \gamma)$, unique up to scalar multiplication, having eigenvalues the same as those of $\phi_{E}$ for small primes. Furthermore, $A=A(z)=\Theta(g)$ has the following properties:

(1) $A \neq 0$ if and only if $E$ has rank zero.

(2) If $e=0$, then $T^{\prime}\left(2^{2}\right) A=\lambda_{2} A$.

(3) If $e=1$, then $T\left(2^{2}\right) A=-\varepsilon_{2} A$.

(4) If $e=2$, then $T\left(2^{2}\right) A=0$.

(5) If $e=3$, then $T\left(2^{2}\right) A(2 z)=0$.

(6) If an odd prime $p$ divides $\gamma$, then $T\left(p^{2}\right) A=A$.

Proof. The proof is mainly by direct computation, but we describe the general method used to establish this result. Let $G=\left\{f_{1}, \ldots, f_{n}\right\}$ be a basis for $V_{0}=$ $\mathcal{M}\left(2^{e} Q, \gamma\right)$. Writing the primes not dividing $2 Q$ in increasing order as $p_{1}, p_{2}, \ldots$, let $V_{i}$ be the $\lambda_{p_{i}}$-eigenspace of $T_{p_{i}}$ in $V_{i-1}$, for $i>0$. If $B_{i}$ is the representation of $T_{p_{i}}$ with respect to $G$, a basis for $V_{i}$ can be found by solving the homogeneous system $\left(\lambda_{p_{i}} I_{n}-B_{i}\right) X=0$. It is clear that each element in such a basis can be taken as a rational linear combination of $G$. By Proposition 2, each space $V_{i}$ is invariant under all operators in $\mathcal{T}$. By direct computation, we have shown that for some $i>0, \operatorname{dim} V_{i}=1$ and so any nonzero element $g \in V_{i}$ is an eigenvector under all $T_{p} \in \mathcal{T}$, with a rational eigenvector in each case.

The first five statements about $A=\Theta(g)$ are also established computationally by calculating sufficiently many terms in the $q$-expansion of $A$. (Note that statement 5 says that each term of even exponent in that $q$-expansion has coefficient 0.) Statement 6 is shown without computation by establishing a one-to-one correspondence between solutions of $f(x, y, z)=n$ and of $f(x, y, z)=p^{2} n$ if $f$ is a ternary form for which the genus symbol $(f / p)$ is equal to $-\left(2^{s} / p\right)$ (which is the case if $\left.p \mid \gamma\right)$. The details are omitted.

Remarks. We used published [4] and unpublished [6] tables of Cremona for the elliptic curves of conductor less than 2000. The invariants $\varepsilon_{p}$ and $\lambda_{p}$ are easily calculated in terms of $E$-note in particular that since $\lambda_{p}=p+1-\left|E_{p}\right|$ [4], it follows that $\left|\lambda_{p}\right|<p+1$ and thus that any eigenvector $g$ obtained by this process must be a cusp vector. A basis $G$ for $\mathcal{M}(N, \gamma)$ can be computed using the process outlined in [13]. The representation matrices $B_{i}$ are found using an algorithm adapted from [1].

In general, the cusp form $A=\Theta(g)$ is, as stated in the introduction, merely a candidate for being a preimage of $\phi_{E}$ under the Shimura correspondence. But in any specific case, the remaining details could, in principle, be tested as follows: One could check by sufficient computation that $A$ is an eigenform under $T\left(p^{2}\right)$ for any odd prime $p \mid N$ for which $p \nmid \gamma$. (Preliminary computations in our examples give strong evidence that $T\left(p^{2}\right) A=-A$ for each such prime.) If so, then $A$ must be sent to some weight two newform $\psi$ by the Shimura correspondence. If for sufficiently many primes, the eigenvalues of $A$ agree with those of $\phi_{E}$, it follows that $\psi=\phi_{E}$ [15]. In each of our examples, we have checked that the eigenvalues of $A$ are the same as those of $\phi_{E}$ for all primes $p<50$ for which $p \nmid N$.

We do not offer a proof that there is always some $i>0$ for which $\operatorname{dim} V_{i}=1$ (that is, for $N>2000$ ). However, we note that results of Gross [9] when $N$ is prime, and Böcherer and Schulze-Pillot [2] when $N$ is squarefree indicate that a preimage 
of $\phi_{E}$ under the Shimura correspondence is a linear combination of the theta series associated to ternary forms if and only if for the $L$-series of $\phi_{E}, L\left(\phi_{E}, 1\right) \neq 0$. This is conjectured to be the case if and only if the rank of $E$ is zero.

The particular choices of the level, discriminant, and genus symbols of the ternary forms which we associate to an elliptic curve $E$ are based on extensive computation of all rational eigenvectors in spaces of ternary forms. By Proposition 1, there are only finitely many possible rational eigenvalues under a given Hecke operator $T_{p}$ on a space of ternary forms $\mathcal{M}$. We have carried out a complete computation of all rational eigenvectors in a large number of spaces, and we present some results in the following:

Theorem 2. Let $N=2^{e} Q$ with $Q$ odd and squarefree and $0 \leq e \leq 3$ as above. For each prime $p$ dividing $N$, let $\varepsilon_{p}$ be chosen as \pm 1 such that $\prod \varepsilon_{p}=-1$, and let $\gamma$ be the genus number associated to the collection $\left\{\varepsilon_{p}\right\}$. Let $\mathcal{S}(N, \gamma)$ be the space of cusp vectors generated by the genus of ternary forms as defined preceding Theorem 1. If $D$ divides $N$, let $n(D, \gamma)$ be the number of weight two newforms of level $D$ and genus number $\gamma$. (If $\gamma \nmid D$, then $n(D, \gamma)=0$ by definition.) Then for all $N<1000$,

(1) $\operatorname{dim} \mathcal{S}(N, \gamma)=\sum_{D \mid N} n(D, \gamma)$

(2) There is a one-to-one correspondence between independent rational eigenvectors in $\mathcal{S}(N, \gamma)$ and isogeny classes of elliptic curves of even rank, conductor $D$ dividing $N$, and genus number $\gamma$.

(3) If $g$ is a rational eigenvector in $\mathcal{S}(N, \gamma)$, but $g$ does not arise from an elliptic curve of conductor $N$ as in Theorem 1, then there is at least one prime $p \mid N$ for which $\Theta(g)$ is not an eigenform under $T\left(p^{2}\right)$.

Remark. The proof of Theorem 2 is again by direct computation. Statement 1 is proved by comparing our tables of ternary forms with tables of $W$-splits of weight two modular forms compiled by Cremona in an unpublished table [5].

\section{EXAMPLE}

We conclude by presenting an example to illustrate the results of Theorems 1 and 2. This example is fairly typical, although in many cases, the space of ternary forms which we must consider has a substantially larger dimension than in this case.

Let $E$ be given by $y^{2}=x^{3}-x^{2}+25158 x-775719$, an elliptic curve of rank 0 and conductor $924=2^{2} \cdot 3 \cdot 7 \cdot 11$, denoted as curve $924 \mathrm{~A} 1$ in [4]. By counting points on $E$ reduced modulo various primes, we find that $\varepsilon_{3}=1, \varepsilon_{7}=1, \varepsilon_{11}=1, \lambda_{5}=-3$, $\lambda_{13}=1$, etc. Since the rank of $E$ is even, we may assume that $\varepsilon_{2}=-\varepsilon_{3} \varepsilon_{7} \varepsilon_{11}=-1$, so the genus number of $E$ is 2 . (These values are confirmed by Table 3 in [4].)

Writing $N=924$ as $2^{e} Q$ with $e=2$ and $Q=231$, we associate to $E$ the genus of ternary forms having level $2^{3} Q=1848$, discriminant $2^{4} Q^{2}=853776$, and genus symbols $(f / 3)=\varepsilon_{3}\left(-2^{4} / 3\right)=-1,(f / 7)=-1$, and $(f / 11)=-1$. Using the methods of [13], we find that there are ten classes of ternary forms in this genus, having representatives $f_{1}, \ldots, f_{10}$ as in the following table. Let $\mathcal{M}(924,2)$ be the $\mathbb{C}$-vector space spanned by these ten forms.

Applying $T_{5}$ to $\mathcal{M}(924,2)$, we find that the -3 -eigenspace, $V_{1}$, has dimension 2 , but in $V_{1}$, the 1-eigenspace of $T_{13}, V_{2}$, is one-dimensional. For some vector in $V_{2}$ 
TABLE 1. $\mathcal{M}(924,2)$ is spanned by the ternary forms $f_{1}, \ldots, f_{10}$. There are seven rational eigenvectors in $\mathcal{S}(924,2)$, each presented as a linear combination of the $f_{i}$ 's. (For example, $g_{1}=f_{1}-f_{2}+$ $\left.\cdots+6 f_{10} \cdot\right)$

$\begin{array}{crrrrrrrrrrr} & \frac{f_{1}}{f_{2}} & \frac{f_{2}}{17} & \frac{f_{3}}{21} & \frac{f_{4}}{24} & \frac{f_{5}}{33} & \frac{f_{6}}{35} & \frac{f_{7}}{35} & \frac{f_{8}}{41} & \frac{f_{9}}{54} & \frac{f_{10}}{54} & \\ b: & 77 & 83 & 110 & 83 & 84 & 41 & 66 & 68 & 54 & 62 & \\ c: & 462 & 164 & 110 & 117 & 98 & 164 & 98 & 83 & 77 & 83 & \\ r: & 0 & -52 & -88 & 6 & -84 & 32 & 0 & -20 & 0 & 16 & \\ s: & 0 & -8 & 0 & 12 & 0 & 28 & -28 & -26 & 0 & 36 & \\ t: & 0 & -10 & 0 & 24 & 0 & 14 & 0 & -8 & -24 & 48 & \\ g_{1}: & 1 & -1 & 1 & -1 & 3 & 1 & -1 & -5 & -4 & 6 & 924 \mathrm{~A} \\ g_{2}: & 1 & 0 & 1 & 2 & 1 & -4 & -2 & 0 & 1 & 0 & 154 \mathrm{~B} \\ g_{3}: & 4 & 6 & -1 & -4 & 2 & -6 & 6 & 0 & -1 & -6 & 132 \mathrm{~B} \\ g_{4}: & 2 & -2 & 2 & -2 & -1 & 2 & -2 & 4 & -1 & -2 & 84 \mathrm{~B} \\ g_{5}: & 1 & 0 & -1 & 2 & -1 & 0 & 0 & 0 & -1 & 0 & 66 \mathrm{~B} \\ g_{6}: & 1 & 4 & 1 & -1 & -2 & 1 & -1 & -5 & 1 & 1 & 44 \mathrm{~A} \\ g_{7}: & 1 & -4 & 1 & 2 & 1 & 4 & 2 & -4 & 1 & -4 & 42 \mathrm{~A}\end{array}$

( $g_{1}$ in the table above), we calculate that

$$
A_{1}=\Theta\left(g_{1}\right)=q^{6}-q^{17}+q^{21}+3 q^{33}-4 q^{41}+\ldots,
$$

a cusp form of weight $3 / 2$, level 1848 , and trivial character. Forms in this space are equal if their $q$-expansions are identical to the term of $q^{577}$ [15], so by direct calculation, we verify that $T\left(2^{2}\right) A_{1}=0$ and $T\left(p^{2}\right) A_{1}=-A_{1}$ for $p=3,7$, and 11 .

In $\mathcal{S}(924,2)$, there are seven independent rational eigenvectors, listed as $g_{1}, \ldots, g_{7}$ in the table above. By computing their eigenvalues for primes $p<50$ which do not divide 924, it appears that there is a correspondence between these eigenforms and the isogeny classes of elliptic curves listed at the right of the table. These are all the elliptic curves of conductor dividing 924 which have genus number 2 [4]. However, if we let $A_{i}=\Theta\left(g_{i}\right)$ for $1 \leq i \leq 7$, we easily check that $A_{2}, A_{5}$, and $A_{7}$ are not eigenforms under $T\left(2^{2}\right) ; A_{2}$ and $A_{6}$ are not eigenforms under $T\left(3^{2}\right) ; A_{3}, A_{5}$, and $A_{6}$ are not eigenforms under $T\left(7^{2}\right)$; and $A_{4}$ and $A_{7}$ are not eigenforms under $T\left(11^{2}\right)$.

\section{REFERENCES}

1. B. J. Birch, Hecke actions on classes of ternary quadratic forms, Computational Number Theory, Walter de Gruyter \& Co., Berlin, 1991, pp. 191-212. MR 93i:11047

2. S. Böcherer and R. Schulze-Pillot, The Dirichlet series of Koecher and Maass and modular forms of weight 3/2, Math. Z. 209 (1992), 273-287. MR 93b:11053

3. M. Bungert, Construction of a cuspform of weight 3/2, Arch. Math. 60 (1993), 530-534. MR 94f: 11035

4. J. E. Cremona, Algorithms for Modular Elliptic Curves, Cambridge University Press, Cambridge, England, 1992. MR 93m:11053

5. _ Dimensions and $W$-splits of cusp forms of level $N \leq 1000$, Unpublished table (1990).

6. Modular elliptic curves: conductors from 1001 to 2000, Unpublished table (1993).

7. M. Eichler, Quadratische Formen und orthogonale Gruppen, Springer-Verlag, Berlin, 1952. MR 14:540a

8. G. Frey, Der Rang der Lösungen von $y^{2}=x^{3} \pm p^{3}$ über $\mathbb{Q}$, Manuscripta Math. 48 (1984), 71-101. MR 86g:11033 
9. B. H. Gross, Heights and the special values of L-series, Number Theory, Montreal 1985 (CMS Conf. Proc.), vol. 7, Amer. Math. Soc., Providence, 1987, pp. 115-187. MR 89c:11082

10. N. Koblitz, Introduction to Elliptic Curves and Modular Forms, Springer-Verlag, New York, 1984. MR 86c: 11040

11. W. Kohnen, Newforms of half-integral weight, J. reine angew. Math. 333 (1982), 32-72. MR 84b: 10038

12. L. Lehman, Rational points on elliptic curves with complex multiplication by the ring of integers in $\mathbb{Q}(\sqrt{-7})$, J. Number Theory 27 (3) (1987), 253-272. MR 89a:11059

13. L Levels of positive definite ternary quadratic forms, Math. Comp. 58 (197) (1992), 399-417. MR 92f: 11057

14. P. Ponomarev, Ternary quadratic forms and Shimura's correspondence, Nagoya Math. J. 81 (1981), 123-151. MR 83a:10043

15. B. Schoeneberg, Elliptic Modular Functions, An Introduction, Springer-Verlag, New York, 1974. MR 54:236

16. R. Schulze-Pillot, Thetareihen positiv definiter quadratischer Formen, Invent. Math. $\mathbf{7 5}$ (1984), 283-299. MR 86d:11042

17. G. Shimura, On modular forms of half-integral weight, Ann. of Math. 97 (1973), 440-481. MR 48:10989

18. H. P. F. Swinnerton-Dyer and B. J. Birch, Elliptic curves and modular functions, Lecture Notes in Mathematics, vol. 476, Springer-Verlag, Berlin, 1975. MR 52:5685

19. J. B. Tunnell, A classical Diophantine problem and modular forms of weight 3/2, Invent. Math. 72 (1983), 323-334. MR 85d:11046

Department of Mathematics, Mary Washington College, Fredericksburg, Virginia 22401

E-mail address: llehman@mwc.edu 